\title{
THE JOVIAN AURORA: ELECTRON OR ION PRECIPITATION?
}

\author{
J. H. Waite, Jr., J. T. Clarke, and. T. E. Cravens
}

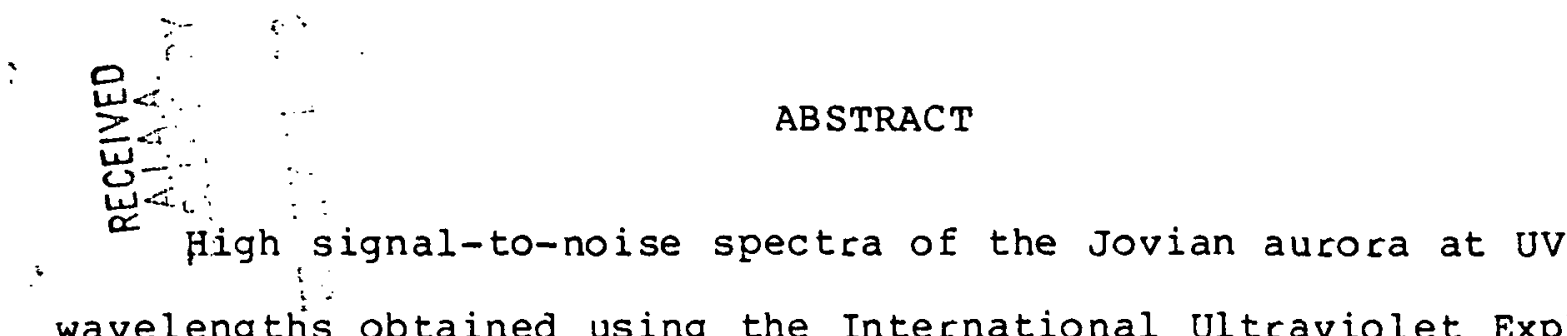
wavelengths obtained using the International Ultraviolet Explorer Observatory (including the brightest Jovian aurora observed to date) set stringent upper limits for sulfur and oxygen emissions, which would be associated with the precipitation of energetic heavy ions in the upper Jovian atmosphere if they were solely responsible for Jovian auroral processes. Model calculations of heavy ion precipitation and corresponding estimates of the associated sulfur and oxygen UV emissions carried out in the preceding companion paper of Horanyi et al. suggest emission values for $1304 \AA$ OI emission that are at least 30 times larger than the upper limit values set by the IUE observations reported here. On the other hand the observed (feature of SII at $1256 \AA$ of $2 \mathrm{kR}$ ) is quite comparable to the theoretically predicted emission intensity. Taken together these observations and calculations suggest that electron as well as ion precipitation play a cole in Jovian auroral processes. In Iight of earlier $X$ ray observations and in situ plasma observations that suggest energetic heavy ion precipitation in the Jovian auroral zone, we suggest a scenario where heavy ion auroral energy deposition is concentrated at altitudes below the homopause (i.e., 100-300 keV/nucleon). Electrons with energies 
. i

of 10 to $30 \mathrm{keV}$ are responsible for the bulk of the observable UV and EUV emissions since they deposit their energy above the methane absorbing layer defined by the homopause. 


\section{INTRODUCTION}

The first indications of $\mathrm{H}_{2}$ Lyman band emission in the Jovian atmosphere were recorded in the sounding rocket spectra of Rottman et al. [1973] and Giles et al. [1976]. However, the first positive identification of Jovian auroral processes was provided by the Voyager ultraviolet spectrometers [Broadfoot et al., 1979; Sandel et al., 1979] and the Intecnational Ultraviolet Explorer (IUE) Observatory [Clarke et al., 1980; Yung et al.' 1982]. The Voyager ultraviolet spectrometer (UVS) experiment measured approximately $60 \mathrm{k}$ Rayleigh $(\mathrm{kR})$ of $\mathrm{H}$ Ly $\alpha$ and about 80 $k R$ total of Lyman and Werner band emission, assuming the auroral zone had a latitudinal extent of $6000 \mathrm{~km}$. If the precipitating particles are electrons [Waite et al., 1983; Yung et al., 1982; Broadfoot et al., 1981; Gerard and Singh, 1982J, this emission level corresponds to a minimum energy flux in the auroral zone of 10 erg $\mathrm{cm}^{-2} \mathrm{~s}^{-1}$, with a total power output exceeding $1-2 \times 10^{13} \mathrm{~W}$. Both the latitude of the observed auroral emissions and the Voyager in situ particle measurements suggest that the Io plasma torus is involved in the generation of energetic particles responsible for the auroral emissions [Broadfoot et al., 1981; Bridge et al., 1979; Krimigis et al.., 1979; Vogt et al., 1979; Gehrels et al.' 1981]. The earlier magnetospheric studies of wave-particle scattering needed to induce auroral precipitation concentrated on energetic electrons [e.g., Waite et al., 1983]. The identified sources of energetic electron scattering however appear to be somewhat inadequate to explain the inferred auroral power budget. Goertz [1980] suggested proton precipitation and 
Thorne [1981, 1983] considered heavy ion precipitation owing to the measured heavy ion composition of the Io torus region.

Credence of the scenario for heavy ion precipitation improved when Gehrels and Stone [1983] reported that measurements of the radial distribution of 1 to $20 \mathrm{Mev} /$ nucleon oxygen, sodium, and sulfur ions in the Jovian magnetosphere suggested pitch angle scattering into the loss cone and subsequent precipitation loss of these ions at a rate comparable to the strong pitch angle diffusion limit. However, none of the plasma waves required for the scattering have yet been identified, and the required precipitating energy flux can only be provided by performing a best-case extrapolation of the Gehrels and Stone [1983] data to the crucial keV energy range, which was not adequately measured by the Voyager spacecraft.

Additional evidence in favor of ion as opposed to electron precipitation is provided by $X$ cay observations of the Jovian aurora by the Einstein X Ray Observatory [Metzger et al.' 1983]. This evidence is based on the unreasonable large energy requirements for $\mathrm{X}$ ray excitation via electron Bremsstrahlung $\left(10^{15}\right.$ to $\left.10^{16} \mathrm{~W}\right)$ as opposed to $\underline{K}$ shell excitation of heavy ions $\left(10^{13}\right.$ to $\left.10^{14} \mathrm{~W}\right)$. We regard these data and their interpretation as strong evidence for ion aurora on Jupiter.

In light of the evidence for heavy ion auroral precipitation, a model of heavy ion aurora and its aeronomical implications was constructed. The results presented in the companion paper by Horanyi et al. [1987] suggest that the observed auroral $\mathrm{H}$ Ly $\alpha$ and $\mathrm{H}_{2}$ Lyman and Werner band emissions 
can be produced by heavy ions with an incident energy flux of 18 erg $\mathrm{cm}^{-2} \mathrm{~s}^{-1}$, which requires an extrapolation of the Gehrels and Stone magnetospheric heavy ion distribution down to an energy of $30 \mathrm{keV} / \mathrm{nucleon}$. Furthermore, these incident heavy ions would be expected to emit characteristic O I, O II, O III and S I, S II, S III recombination emissions that should be observable to ultraviolet spectrometers such as IUE.

To test this hypothesis, long-time exposure measurements of the Jovian aurora with high signal-to-noise ratio in the wavelength range of 1200 to 1500 \& were obtained using the IUE Observatory. The results of these measurements and subsequent spectral modeling presented here show mixed evidence of the oxygen and sulfur recombination emissions that would be expected if heavy ions produced the ultraviolet emissions. We note that only the UV emissions which are produced above the UV absorbing hydrocarbon layer are observed by the IUE and Voyager ultraviolet spectrome ters.

This evidence combined with the recent IUE [Skinnes et al., 1984] and Voyager [Herbert et al., 1986] observations of the longitudinal distribution of the Jovian UV aurora [Skinner et al., 1984] suggests to us that electrons as well as ions play a role in Jovian auroral processes. Furthermore, we conclude that the energetic heavy ions observed by Gehrels and Stone [1983] may indeed be responsible for the $x$ ray emissions, but are probably confined in a sufficiently high energy range ( $300 \mathrm{keV})$ that they deposit the bulk of their energy below the methane homopause. Such a scenario is similar to that of terrestrial aurora, where 
both energetic precipitating electrons and protons are known to participate in auroral processes.

\section{OBSERVATIONS}

Two spectra of the Jovian aurora at wavelengths between 1150 and 1950 \& were obtained using the IUE Observatory on July 12, 1985, from 0300 to 1100 UT. The IUE low dispersion mode was used and the image exposure time was set at $75 \mathrm{~min}$ to maximize the signal-to-noise ratio in the $\mathrm{H}_{2}$ band systems between 1250 and $1500 \AA$. As a result the spectrum above 1500 \& and the $H$ Ly $\alpha$ emission feature were intentionally over exposed, and could not be used in determining the strength of the observed aurora. An image of the northern auroral zone and a second image of the southern auroral zone were obtained. Proper time aliasing was carried out to provide System III longitude coverage which is consistent with maximum polar zone exposure las a result of the Jovian magnetic axis tilt of $10^{\circ}$, Clarke et al., 1980] and also to avoid observing along a line of sight through the Io torus (i.e., when the torus extended either above or below the line of sight of the auroral zone). In spite of this geometrical correction the observed northern auroral zone was found to be over a factor of 3 brighter than the southern auroral zone during the observation period. For this reason analysis of the 1985 IUE data has concentrated on the northern Jovian auroral zone image SWP 26401. Additional images were obtained in a second observing campaign on August 5, 1986 and a third campaign on December 14, 1986. The auroral intensity was moderate and the corresponding 
signal-to-noise ratio was moderate as well. These spectra. of the north and south auroral zone, SWP 28845, SWP 28847, and SWP 29879 are consistent with the SWP 26401 spectra and are also presented herein.

The data were acquired by centering the IUE short wavelength spectrograph (9" $x$ 20" field of view) on the respective pole in the north-south direction and tracking the longitude of maximum emission in the east-west direction ( $\lambda_{\text {III }}=180^{\circ}$ at the north pole and $\lambda_{\text {III }}=0^{\circ}$ at the south pole). Using the spatial imaging capabilities of the IUE spectrograph, all of the auroral emissions were found to be within the central $9^{\prime \prime} \times 15^{\prime \prime}$ rectangular portion of the aperture. Auroral spectra for each exposure were formed by summing the data from those portions of the aperture that contained auroral emissions. Other Jovian spectra containing no auroral emissions were used to remove the non-auroral planetary signal and instrumental effects such as gratingscattered light. Uncertainties in the level of the background signal are small compared to the auroral signal and produce a negligible uncertainty in the images, which is shown in Figure 1. This auroral spectrum is only accurate between 1225 and 1500 \& because of the long exposure time of $75 \mathrm{~min}$ used in the observation. However, the spectrum in this wavelength region for the northern auroral zone has a higher signal-to-noise ratio than was obtained by summing the brightest archived short-time exposure images of the Jovian aurora. Therefore, the analysis that follows in the next section will concentrate on images SWP 26401 and SWP 29879 the best northern and southern auroral zone spectra, respectively. 


\section{ANALYSIS}

A visual inspection of the northern auroral spectrum SWP26401 (see Figure 1, top panel) shows no emission near $1304 \AA$, where one would expect the O I triplet if oxygen ions were precipitating above the level of the methane homopause. Analysis of the possible features present in the spectra gives an upper limit for the $\mathrm{O} I$ emission of $\sim 160 \mathrm{R}$.

Other heavy ion features in the spectrum are blended in with $\mathrm{H}_{2}$ band features. Such is the case for the expected $\mathrm{S}$ II emission line at 1256 A. The presence of these emission features can only be determined by carefully subtracting the expected $\mathrm{H}_{2}$ band emissions from the observed spectrum. This subtraction was carried out using the measured laboratory $\mathrm{H}_{2}$ band spectra of Ajello et al. [1984] corrected for atmospheric absorption. Measured spectra with electron energies of 20 and $100 \mathrm{eV}$ were used and have been degraded to the resolution of the IUE spectra using a triangular spectrometer response function. The relative contribution of each spectrum was determined by searching parameter space for the best fit to the IUE spectra, which occurred at a $20 / 100 \mathrm{eV}$ cross section ratio of 0.54 .

Radiative transfer effects, particularly those due to hydrocarbon absorption, were modeled by using the transmission curves of Yung et al. [1982] for various particle penetration depths. The effects of hydrocarbon absorption due to $\mathrm{CH}_{4}$ and $\mathrm{C}_{2} \mathrm{H}_{2}$ were examined by systematically multiplying the laboratory spectra by the transmission curves of Yung et al. at each 
respective wavelength in the spectra. Each transmission case (aE) was then adjusted in intensity until a minimum deviation between the adjusted laboratory and IUE spectra was obtained. The six different transmission cases correspond to differences in incident electron energy, or equivalently, depth of penetration of the precipitating particles into the atmosphere. The results of these six cases can then be independently examined for relative fit. The fit indicates that electrons between 10 and 30 $\mathrm{keV}$ correspond to the level of auroral particle penetration with a best fit for an electron primary precipitation energy of 22.5 kev, or equivalently particles precipitating to an atmospheric density of $4 \times 10^{10}$ to $5 \times 10^{13} \mathrm{~cm}^{-3}$ (just above the level of the methane homopause). This result is consistent with the earlier findings of Yung et al. [1982] with regard to the level of atmospheric excitation.

This transmission study thereby allows us to obtain a best fit to the "adjusted" laboratory spectra. These adjusted spectra were then subtracted from the IUE spectra and the resulting difference is shown in the bottom panel of each figure. Statistical error bars for the differenced data are also shown. No oxygen ion emission features are observed above the level of statistical error. A (l sigma) feature just above the statistical error bar exists at $1256 \AA$. However, there is no clear indication that the feature is real and not simply an artifact of the fit to the $\mathrm{H}_{2}$ emission spectrum.

A blowup of the difference spectrum for the critical wavelength range from 1240 to 1320 \& is shown in Figure 2 . 


\section{(1. ल.}

Although no emission features are seen above the statistical uncertainty level, no feature near $1304 \AA$ is visible but a small feature near 1256 \&ay be seen. This feature is in a region of rapidly changing $\mathrm{H}_{2}$ emission cross section and is subject to uncertainties in the assumed spectrometer response function. Only 5 to $10 \%$ uncertainty in the $\mathrm{H}_{2}$ emission model would be needed to explain the feature. However, its singular appearance in the difference spectra and its exact correspondence to the 1256. A wavelength suggest a 1 sigma SII emission feature. Analysis of the emission that could be present in these features is $160 \mathrm{R}$ for $1304 \&$ and $2400 \mathrm{R}$ for 1256 where these numbers have been corrected for hydrocarbon absorption effects. However, it must be kept in mind that these features represent only upper limits to features that are just visible above the statistical noise level.

Additional analysis of images SWP28845, SWP 28847, and SWP29879 was also carried out. Similar cross section ratios and transmission features again provided a good fit to the spectra. The only necessary change was to scale by the change in the auroral intensity as determined by integrating the overall flux from 1240 to $1480 \AA$. The results of comparing these spectra with $\mathrm{H}_{2} \mathrm{lab}$ spectra are shown in Figures 3,4 , and 5 . There is no indication of sulfur or oxygen emission features in the lower signal to noise spectra of SWP28845 and SWP28847. However, in spectra SWP29879 (Figure 5) there is again a feature at 1256 A The feature is more statistically significant ( 2 sigma) in this case since the difference at $1256 \AA$ is almost as large $(2000 \mathrm{R})$, 
but the overall auroral brightness is a factor of 2.5 to 3.0 less intense than the SWP26401 spectra. In this case 15 to 308 errors in the $\mathrm{H}_{2}$ modeling are required to explain the feature. Again there is no indication of emission at $1304 \&(<150 \mathrm{R})$.

\section{DISCUSSION}

The upper limits of heavy ion emission features in the measured IUE spectra must be compared to those predicted by heavy ion auroral modeling, which are presented in the companion paper by Horanyi et al. [1987]. To understand the implications of these constraints on the heavy ion emissions one must first understand the model calculations.

Theoretical calculations of the energy deposition of energetic oxygen ions precipitating into the Jovian atmosphere show that an input power of $10-20 \mathrm{erg} \mathrm{cm}^{-2} \mathrm{~s}^{-1}\left(\sim 2 \times 10^{13} \mathrm{~W}\right)$ is required to produce the $80 \mathrm{kR}$ of Lyman and Werner band emissions observed by the Voyager UVS experiment. The aeronomical effect of this energetic ion precipitation is very similar to that of energetic electrons [Horanyi et al., 1987; Waite et al., 1983]; that is, excitation, dissociation, and ionization rates are very similar for both types of particle precipitation.

Comparison of the model and observations at $1304 \AA$ is definitive. The absence of emission features of $\mathrm{H}_{2}$ in this spectral region allows tight constraints to be placed on the observed spectra; less than 160 R of emission are present near 1304 A. Theoretically the emission is expected to be produced by 
direct excitation of $O I$. Although cross sections for this process have not been measured, they can be estimated at high ion energies. The electron and ion cross sections should be about the same at equal velocities, according to atomic scattering theory. This conclusion, however, is only valid at high energies. We have estimated the 1304 \& emission intensity using the electron excitation cross sections of Vaughan and Doering [1986] and the electron on O ionization measurements of Fite and Brackmann [1959]. The fraction of electron ionizations that leads to excitation of the ${ }^{3} \mathrm{~s}^{0}$ excited state required for 1304 \& emission, and at sufficiently high energies for the Born approximation to hold $(200 \mathrm{eV})$, is $4.2 \%$. Using this efficiency and the total column production of $O$ I stripping reactions from the Horanyi et al. model (presented in the companion paper), we can estimate the expected 1304 \& emission. The O I 1304 \& intensity calculated by this method gives $5 \mathrm{kR}$ for a $26 \mathrm{erg} \mathrm{cm}^{-2}$ $\mathrm{s}^{-1}$ incident ion power (energy flux scaled to the intensity of the bright aurora of SWP26401). Five $k R$ is a factor of 30 larger than the upper limit of the IUE measurement. This suggests that oxygen ions are not responsible for the production of the bulk auroral UV emissions seen by IUE.

The situation at 1256 \& SI is quite different from a theoretical point of view since the expected excitation comes from the excess energy associated with charge transfer and stripping collisions. In this case there is a significant difference between electron and ion precipitation as demonstrated by our calculations [Horanyi et al., 1986] 108 of the incident 
energy goes into excitation and excess kinetic energy associated with charge transfer and stripping collisions of oxygen ions and the target hydrogen molecules. The percent energy lost to these processes for energetic precipitating sulfur ions is probably also close to 108 , although calculations for sulfur precipitation were not carried out. This energy is primarily lost to the precipitating ions during stripping collisions and does not include the energy carried off by the electrons produced in this process. This energy is made available during: charge exchange collisions as kinetic and internal energy of the product $0^{+q}$ and $\mathrm{H}_{2}^{+}$ions :

$$
\left.\mathrm{O}^{+(\mathrm{q}+\mathrm{I})}+\mathrm{H}_{2} \rightarrow \mathrm{O}^{+\mathrm{q}}+\mathrm{H}_{2}^{+{ }^{*}}{ }^{*}\right)
$$

Some of the $\mathrm{H}_{2}^{+}\left(^{*}\right)$ might dissociate to give $\mathrm{H}\left({ }^{*}\right)+\mathrm{H}^{+}$.

Laboratory data and theoretical calculations for these types of processes in general indicate that about half of the available energy goes into kinetic energy and half into internal energy (excitation). This gives about $5 \%$ of $26 \mathrm{erg} \mathrm{cm}^{-2} \mathrm{~s}^{-1}$ (the energy flux scaled by comparing the theoretical $\mathrm{H}_{2}$ Lyman and Werner band emisions to the integrated IUE spectra in image SWP 26401) or about $1.3 \mathrm{erg} \mathrm{cm} \mathrm{cm}^{-2} \mathrm{~s}^{-1}$, or $8 \times 10^{11} \mathrm{eV} \mathrm{cm}^{-2} \mathrm{~s}^{-1}$. Suppose that about half of this $\left(4 \times 10^{11} \mathrm{eV} \mathrm{cm}^{-2} \mathrm{~s}^{-1}\right)$ goes to excite $0^{+q}$ and $\mathrm{S}^{+\mathrm{q}}$ ions and half to produce $\left.\mathrm{H}^{*}\right)+\mathrm{H}^{+}$.

The photon energies for typical S II, S III, S IV and $O$ II, O III, and O IV emission features (S I and O I are not likely to be excited, since the charge transfer reaction is endothermic for 
these charge states) are all roughly $20 \mathrm{ev;}$ thus the total emission rate is $4 \times 10^{11} \mathrm{eV} \mathrm{cm}^{-2} \mathrm{~s}^{-1}$ divided by $20 \mathrm{ev}$, or 20 kR. Taking into account the prominent emission features of $S I$, S II, S III, S IV, O I, O II, and O III, one gets a lower limit for the predicted intensity of about $2 \mathrm{kR}$ per feature. Such features have not been reported in the auroral spectra from Voyager, although the same features are present in the Io plasma torus and might obscure the auroral emissions. One such feature that can be studied with IUE is the S II Iine at $1256 \AA$. In the case of the SWP26401 and SWP29879 about 2 kR of emission at 1256 A was observed with IUE when proper corrections are made for atmospheric transmission. This is quite close to the theoretically predicted emission. Yet the reader can clearly see that there are large uncertainties in the presently estimated theoretical emission cross sections. In addition uncertainties in modeling the $\mathrm{H}_{2}$ band spectra affect the analysis of the $1256 \AA$ feature while $1304 \AA$ is located in a minimum in the $\mathrm{H}_{2}$ band systems and is not affected by $\mathrm{H}_{2}$ emission modeling. Obviously, better observations of this emission feature and laboratory measurements of the ion stripping production of excited state $S$ II are needed before more definitive results are possible for this emission.

The IUE observations are therefore somewhat ambigious on the role of ion precipitation in the Jovian auroca. On the one hand the absence of emission from OI at $1304 \AA$ suggests little contribution to the observable UV aurora from ion precipitation, and on the other hand the observed emission feature (?) of SII at 
1256 \& of $2 \mathrm{kR}$ is close to that predicted by simple theory. Clearly there are significant uncertainties in the estimates of cross sections for both the OI 1304 \& and SII 1256 \& emission processes. Furthermore, in the case of 1256 \& there is additional uncertainty that arises from the necessary modeling and subtraction of $\mathrm{H}_{2}$ band features in this spectral region. In spite of these uncertainties the observations suggest ion auroral emission at 1256 from SII and no emission from OI at $1304 \AA$.

These two somewhat conflicting pieces of evidence may be partially reconciled by comparing the two excitation processes. In the case of sulfur the excitation process is a consequence of electron stripping followed by exothermic charge transfer.from SIII to SII with enough available energy to leave the SII atom in.: an excited electronic state. The case of OI excitation is quite: different since OII to OI charge transfer with" $\mathrm{H}_{2}$ is an endothermic process and will not result in OI excitation. The OI excitation considered is therefore a direct excitation of neutral oxygen as a result of collisions with $\mathrm{H}_{2}$. The relevant point here is that due to charge state equilibration of the ion beam (see Horanyi et al., 1987, figure 3 ) the tendency for the beam is to be more highly ionized at higher altitudes, thus favoring the electron stripping/charge exchange excitation. Whereas the beam must be neutralized at greater depths in the atmosphere before direct excitation of $O I$ is possible. The net result is that the charge exchange/stripping region SII may be more distributed in altitude and lie several scale heights above the region of or direct excitation. Therefore, if the ions are in general 
energetic enough to deposit the bulk of their energy below the homopause, the SII emission could conceivably lie above the homopause whereas the OI excitation and the bulk of the auroral ion excitation would occur below the homopause. For energetic ion aurora (100 - $300 \mathrm{keV} / \mathrm{nucleon})$ the observed $\mathrm{H}_{2}$ Lyman and Werner band emissions would be less than $10 \mathrm{kR}$. The precipitation of energetic electrons [Waite et al., 1983] would then be required in addition to ion precipitation in order to generate the required $\mathrm{H}_{2}$ intensity.

Several possible sources of energetic electron plasma wave scattering have been identified by Thorne [1983] and Inan [1986]. They include electromagnetic hiss $\left(6 \mathrm{x} \cdot 10^{12} \mathrm{~W}\right)$ [Thorne and Tsurutani, 1979], electromagnetic chorus (.<10.12.W)-[Thorne, 1984; Inan, 1986], and electrostatic $n+1 / 2$ cyclotronrharmonic waves $\left(<10^{12} \mathrm{~W}\right)$ [Thorne, 1983]. These sources seem marginally capable of supplying the power required in energetic electrons to explain the ultraviolet auroral emissions visible above the methane homopause. However, these estimates are based on equatorial measurements and may not be representative of processes at higher latitudes such as plasma waves or double layers that may play a role in determining auroral electron acceleration. Additional evidence that electrons play a role in the ultraviolet aurora can be found in the system III longitude dependence of the UV aurora which was reported by Skinner and Moos [1984] and Herbert et al. [1986]. Although the authors made no definitive stand on whether ion or electron precipitation was responsible for the aurora, we feel that the longitudes where 
maximum UV auroral emission are observed are consistent with the longitudes of energetic electron precipitation calculated by Dessler and Hill [1979]. Although the zones of auroral emission are somewhat larger than the predicted precipitation zones they are well within the uncertainty of the inferred surface magnetic field structure [Acuna et al., 1983]. This evidence taken in conjunction with the IUE observations reported in this paper present a case for energetic electrons (10 to $100 \mathrm{keV}$ ) playing a major role in producing the observable UV aurora at Jupiter. However, evidence for the precipitation of energetic $(>300$ keV nucleon) ions is also quite compelling. The observed radial distribution of such energetic heavy ions by voyager. [Gehrels et al., 1981], interpreted in conjunction with desived values of the radial ion diffusion coefficient by Siscoe and Summers [1981], suggests that sulfur, oxygen, and sodium ions are precipitating into the upper atmosphere at a rate comparable to the strong pitch angle diffusion rate between 6 and $8 \mathrm{R}_{\mathrm{J}}$ [Gehrels and Stone, 19831. Furthermore, the Einstein Observatory $X$ ray measurements provide a fairly strong case for $K$ shell excitation of heavy ions as the source of Jovian auroral $x$ ray emissions. The point of departure in the present paper is the extent of the role of heavy ions in auroral processes. The evidence presented in this paper suggests that only ions above $\sim 100-300 \mathrm{keV} / \mathrm{nucleon}$ precipitate in to the atmosphere. In this case little emission specific to oxygen and sulfur would be expected because the bulk of the energy deposition occurs below the homopause. Eurthermore, the $x$ ray emission power can be accommodated, and there is no need to 
extend the heavy ion energy distribution to lower energies, as was postulated by Gehrels and Stone [1983] and modeled by Horanyi et al. [1987]. For a further discussion of these latter two points, see the companion paper by Horanyi et al. [1987].

\section{CONCLUSIONS}

The suggested scenario for Jovian auroral processes presented here is a case much like that of earth, with energetic heavy ions ( $>300 \mathrm{keV} /$ nucleon) and energetic electrons (10 to 100 keV) both playing a role in auroral processes. The heavy ions precipitate between Jovian invariant latitudes of $66^{\circ}$ and $69^{\circ}$ [Gehrels and Stone, 1983] and deposit most of their:energy below the level of the methane homopause. They produce $x$ ray emissions that can be observed by instruments such as the Einstein Observatory and the planned AXAF Observatory. The power dissipation of these ions is of the order of $10^{13} \mathrm{w}$. The precipitating electrons have energies on the order of 10 to 100 $\mathrm{keV}$ and therefore deposit most of their energy above the methane homopause. They result in the bulk of the $\mathrm{H}_{2} \mathrm{UV}$ emissions that, have been observed by both Voyager and IUE UV spectrometers. Their total energy dissipation in the Jovian atmosphere is 1 to 3 $x 10^{13} \mathrm{~W}$ at an invariant latitude just slightly poleward of the ion $\mathrm{X}$ ray emissions. The spatial location of these UV emissions can and should be carefully observed with spatially resolving instruments like the upcoming Hubble space Telescope (HST).

A further test of the proposed scenario would be to simultaneously observe both the UV and $X$ ray auroral emissions as 
a function of System III longitude. It is expected that the electron precipitation and thus UV emissions would follow the longitudinal pattern suggested by Dessler and Hill [1979], and that the ion precipitation and $x$ ray emissions would be out of phase and fill in the longitudinal gaps of the UV pattern.

Acknowledgments. The authors would like to acknowledge helpful discussions with Alex Dessler and Darrell F. Strobel.. We are also deeply indebted to J. M. Ajello who provided us with the digitized laboratory $\mathrm{H}_{2}$ spectra used in this study. This research would not have been possible without the support of the IUE guest investigator program and the IUE Observatory staff, and the IUE Remote Data Analysis Facility staff at Goddard Space. Flight Center. Partial support for this research comes from the NASA Outer Planet Data Analysis Program and NASA grant NGR 23005-015. 


\section{REF ERENCES}

Acuna, M. H., K. H. Behannon, and J.E.P. Connerney, Jupiter's magnetic field and magnetosphere, in Physics of the Jovian Magnetosphere, edited by A. J. Dessler, p. 1, Cambridge University Press, 1983.

Ajello, J. M., D. Shemansky, T. L. Kwok, and Y. L. Yung, Studies of extreme-ultraviolet emission from Rydberg series of $\mathrm{H}_{2}$ by electron impact, Phys. Rev. A, 29, 636-653, 1984.

Atreya, S. K., Y. L. Yung, T. M. Donahue, and E. S. Barker, Search for Jovian auroral hot spots, Astrophys. J. (Lett.) 218, L83-L87, 1977 .

Bridge, H. S., J. W. Belcher, A. J. Lazarus, J. D. Sullivan, R. L. McNutt, F. Bagenal, J. D. Scudder, E. C: Si.ttler, G. L. Siscoe, V. N. Vasyliunas, C. K. Goertz, and C. M. Yeates, Plasma observations near Jupiter: Initial results from Voyager 1, Science, 204, 987-991, 1979.

Broadfoot, A. L., M. J. S. Belton, P. Z. Takacs, B. R. Sandel, D. E. Shemansky, J. B. Holberg, J. M. Ajello, S. R. Atreya, T. M. Donahue, H. W. Moos, J. L. Bertaux, J. E. Blamont, D. F. Strobel, J. C. McConnell, A. Dalgarno, R. Goody, and M. B. McElroy, Extreme ultraviolet observations from Voyager I encounter with Jupiter, Science, 204, 979-982, 1979.

Broadfoot, A. L., B. R. Sandel, D. E. Shemansky, J. C. McConnell, G. R. Smith, J. B. Holberg, S. K. Atreya, T. M. Donahue, D. F. Strobel, and J. L. Bectaux, Overview of the Voyager ultraviolet spectrometry results through Jupiter encounter, J. Geophys. Res., 86, 8259-8284, 1981. 
Clarke, J. T., H. W. Moos, S. K. Atreya, and A. L. Lane, Observations from Earth orbit and variability of the polar aurora on Jupiter, Astrophys. J. (Lett.), 241, L179-L182, 1980.

Dessler, A. J., and T. W. Hill, Jovian Longitudinal control of Io-related radio emissions, Astrophys. J., 227, 664-675, 1979. Fite, W. L., and R. T. Brackmann, Ionization of atomic oxygen on electron impact, Phys. Rev., 113, 815, 1959.

Gehrels, N., and E. C. Stone, Energetic oxygen and sulfur ions in the Jovian magnetosphere and their contribution to the auroral excitation, J. Geophys. Res., 88, 5537-5550, 1983.

Gehrels, N., E. C. Stone, and J. H. Trainor, Energetic oxygen and sulfur in the Jovian magnetosphere, J. Geophys. Res., 86 , $8906-8918,1981$

Gerard, J. $-\mathrm{Cl}$., and V. Singh, A model of enerqy deposition of energetic electrons and EUV emissions in the Jovian and Saturnian atmospheres and implications, J. Geophys. Res., 87 , $4525-4532,1982$.

Giles, J. W., H. W. Moos, and W. R. Mckinney, The far ultraviolet (1200-1900 A) spectrum of Jupiter obtained with a rocket-borne mutichannel spectrometer, J. Geophys. Res., 81, 5797, 5806, 1976.

Goertz, C. K., Proton aurora on Jupiter's nightside, Geophys. Res. Lett., 7, 365-368, 1980 .

Herbert, F., B. R. Sandel, and A. L. Broadfoot, Observations of: the Jovian UV Aurora by Voyager, J. Geophys. Res., in press, 1986 . 
Horanyi, M., T. E. Cravens, and J. H. Waite, Jr., The precipitation of energetic heavy ions into the upper atmosphere of Jupiter, J. Geophys. Res., companion paper, this issue.

Inan, U. S., Jovian VLE chorus and Io torus aurora, J. Geophys. Res., 91, 4543-4550, 1986.

Krimigis, S. M., T. P.Armstrong, W. S. Axford, C. P. Bostrom, C. Y. Fan, G. Gloeckler, L. J. Lanzerotti, E. P. Kearth, R. D. Zwickl, J. E. Carbary, and D.C.Hamilton, Low-energy charged particle environment at Jupiter: A first look, Science, 204, $998-1003,1979$.

Metzger, A. E., D. A. Gilman, J. L. Luthey, K. C. Hurley, H. W. Schnopper, F. D. Seward, and J. D. Sullivan, The detection of $\mathrm{X}$ rays from Jupiter, J. Geophys. Res., 88, 7731-7741, 1983 . Rottman, G. J., H. W. Moos, and C. S. Freer, The far-ultraviolet spectrum of Jupiter, Astrophys. J. (Lett.), 184, L89, 1973. Sandel, B. R., D. E. Shemansky, A. L. Broadfoot, J. L. Bertaux, J. E. Blamont, M. J. S. Belton, J. M. Ajello, J. B. Holberg, S. K. Atreya, T. M. Donahue, H. W. Moos, D. F. Strobel, J. C. McConnell, A. Dalgarno, R. Goody, M. B. McElroy, and P. Z. Takacs, Extreme ultraviolet observations from Voyager 2 encounter with Jupiter, Science, 206, 962-966, 1979. Siscoe, G. L., and D. Summers, Cenţ̣ifugally driven diffusion of Iogenic plasma, J. Geophys. Res., 86, 8471-8479, 1981. Skinner, T. E., and H. W. Moos, Comparison of the Jovian north and south pole aurorae using the IUE Observatory, Geophys. Res. Lett., 11, 1107-1110, 1984. 
Shemansky, D. E., and G. R. Smith, The Voyager 1 EUV spectrum of the Io plasma torus, J. Geophys. Res., 86, 9179-9192, 1981.

Thorne, R. M., Jovian auroral secondary electrons and the ir influence on the Io plasma torus, Geophys. Res. Lett., 8, 509512,1981 .

Thorne, R. M., Microscopic plasma processes in the Jovian magnetosphere, Chapter 12 in Physics of the Jovian Magnetosphere, edited by A. J. Dessler, Cambridge University Press, 1983.

Thorne, R. M., and B. J. Tsurutani, Diffuse Jovian aurora influenced by plasma injection from Io, Geophys. Res. Lett. ' $6,649,1979$.

Vaughan, S. O., and J. P. Doering, Absolute experimental differential and integral electron cross sections for atomic oxygen: II. The $\left({ }^{3} p->^{3} s^{0}\right)$ transition (1304 A) from 16.5 to 200 eV, J. Geophys. Res., in press, 1986.

Vogt, R. E., W. R. Cook, A. C. Cummings, T. L. Garrard, N. Gehrels, E. C. Stone, J. H. Trainor, A. W. Schardt, T. Conlon, N. Lal, and F. B. McDonald, Voyager 1 : Energetic ions and electrons in the Jovian magnetosphere, Science, 204, 1003$1007,1979$.

Waite, J. H., Jr., T. E. Cravens, J. Kozyra, A. F. Nagy, S. K. Atreya, and R. H. Chen, Electron precipitation and related aeronomy of the Jovian thermosphere and ionosphere, J. Geophys. Res., 88, 6143-6163, 1983. 
Yung, Y. L., G. R. Gladstone, K. M. Chang, J. M. Ajello, and S. K. Srivastava, $\mathrm{H}_{2}$ fluorescence spectrum from 1200 to 1700 \& by electron impact: Laboratory study and application to Jovian aurora, Astrophys. J. (Lett.), 254, L65-L69, 1982. 


\section{FIGURE CAPTIONS}

Fig. 1 The EUV spectrum of the northern Jovian auroral zone obtained on image SWP 26401 with the IUE Observatory between 1240 and $1480 \&$ (solid line). Superimposed upon it is the laboratory $\mathrm{H}_{2}$ spectrum of Ajello et al. [1984] (dashed line) (upper panel). Spectral energy flux is plotted as a function of wavelength. The bottom panel shows the difference spectrum obtained by subtracting the $\mathrm{H}_{2}$ laboratory spectrum from the IUE spectrum. The difference reflects emissions that are not explained by $\mathrm{H}_{2}$ Lyman and Werner band emissions or due to radiative transfer effects not included in the present model.

Fig. 2 Same spectra as shown in Figure 1 , but expanded in the spectral region between 1240 and 1320 \& to give a better indication of this important spectral range where S II $(1256$ A) and $O$ I (1304 A) emissions are expected.

Fig. 3 Same format as Figure 1 but for SWP 28845 of the southern Jovian auroral zone. Note the decrease in auroral intensity .

Fig. 4 Same format as Figure 1 but for SWP 28847 of the northern auroral zone as seen on August 5, 1986, with IUE. Note again the decreased emission intensity and the continued lack of significant emission at 1256 \& and 1304 \&. 
Fig. 5 Same format as Figure 1, but for SWP29879 of the southern auroral zone as seen on December 14, 1987 with IUE. Note the lack of emission at $1304 \AA$ and the small suggested emission feature at $1256 \AA$ in the bottom (difference spectral panel. 

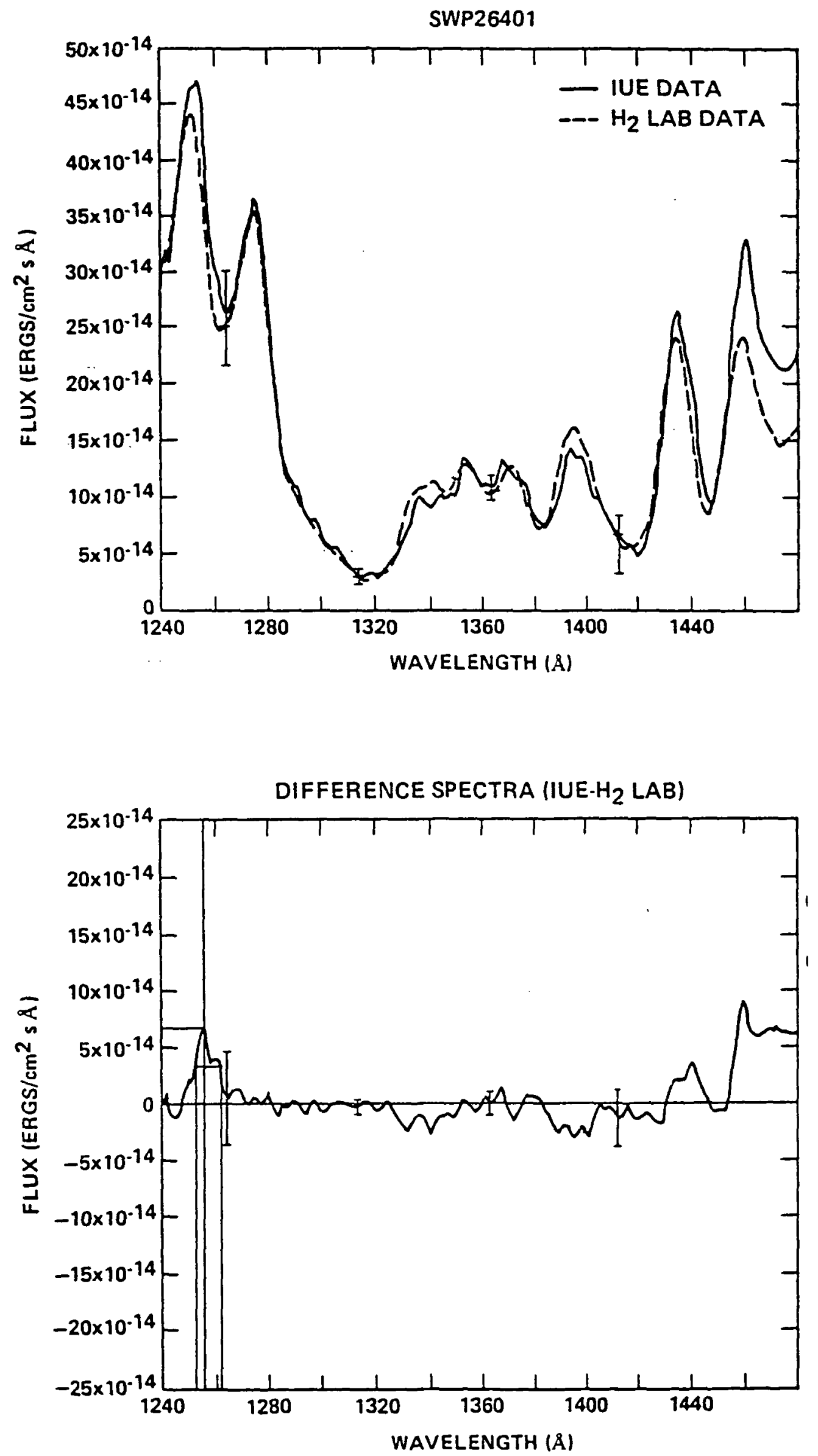

Flaure 1 

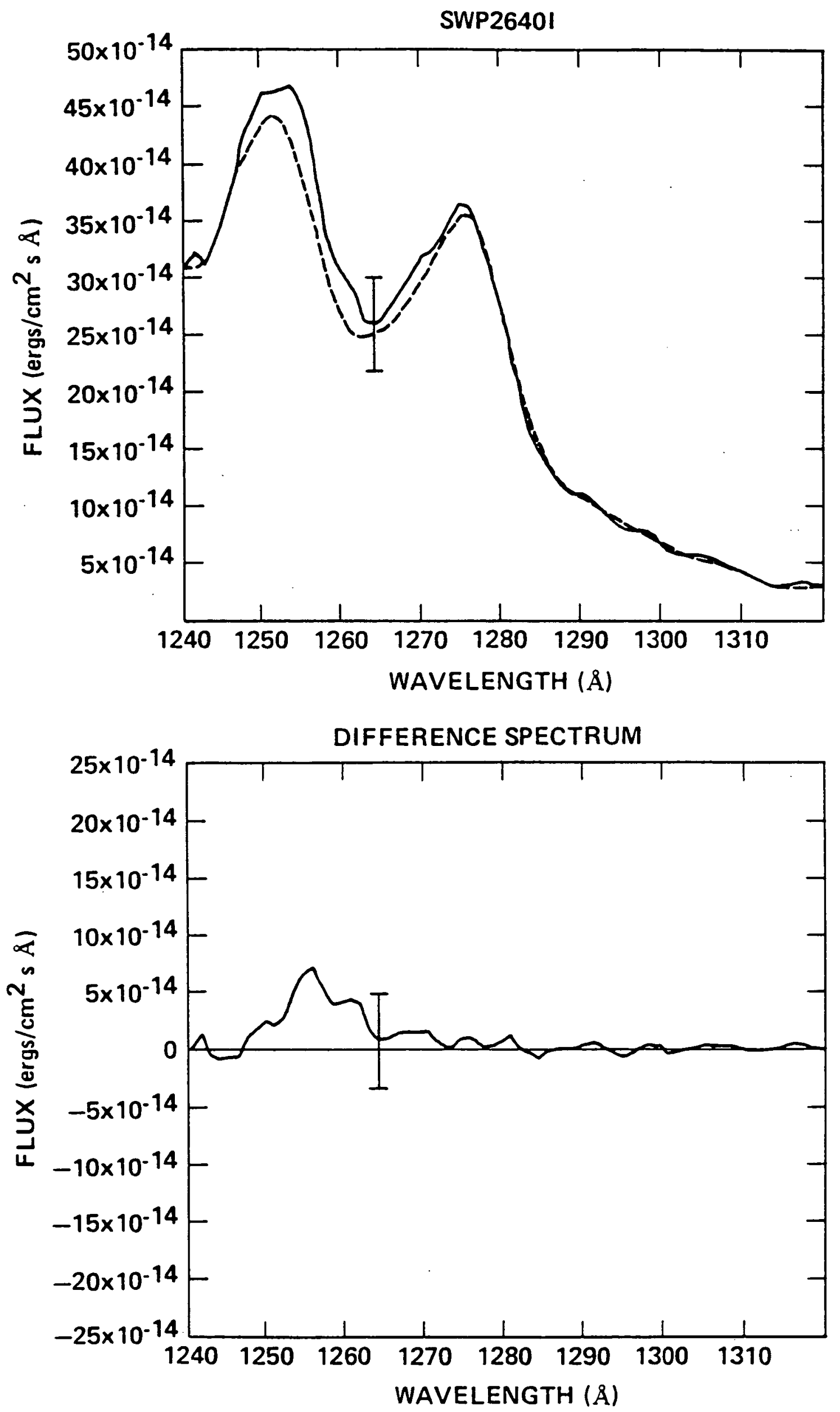

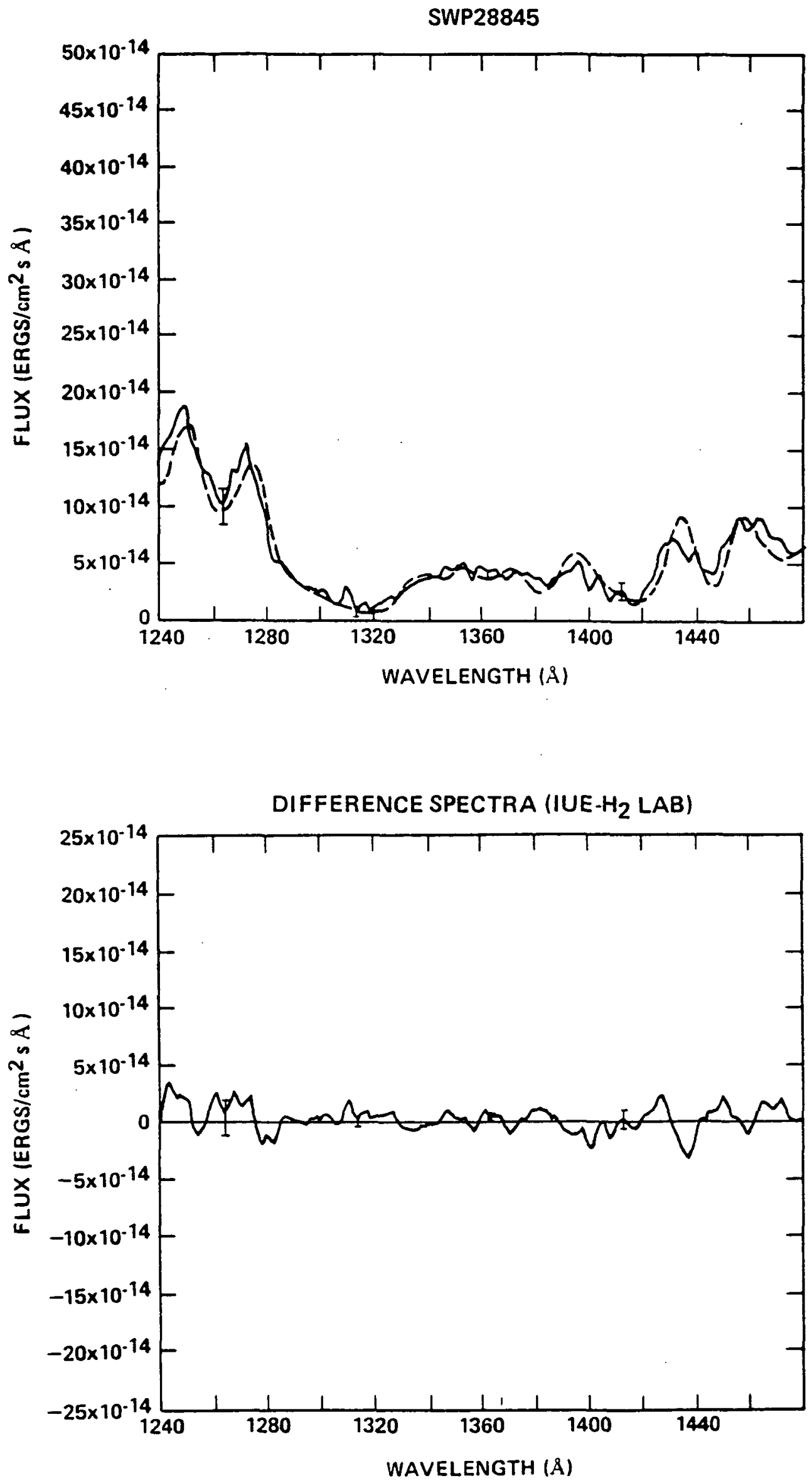

Finure 3 

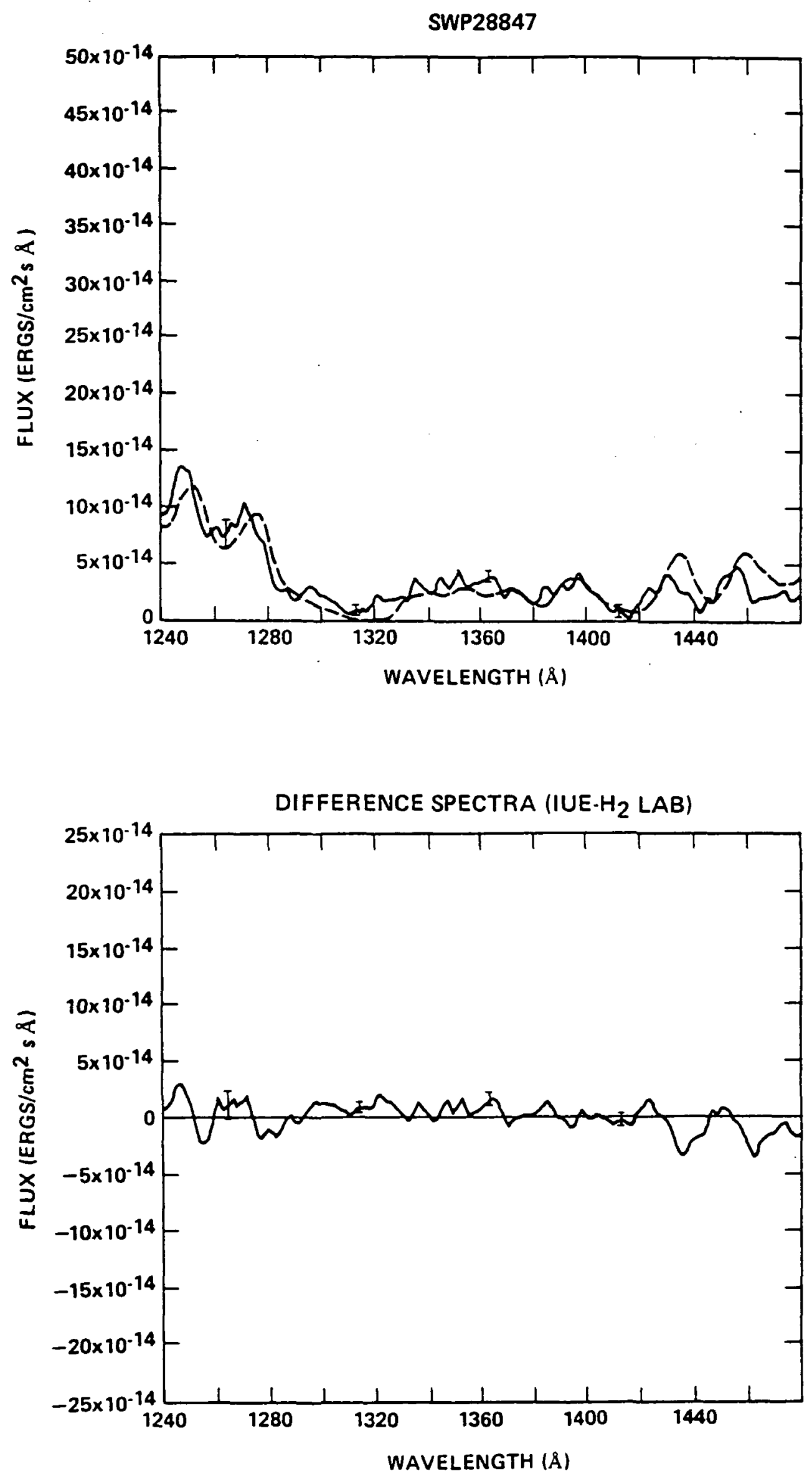

Fiave 4 

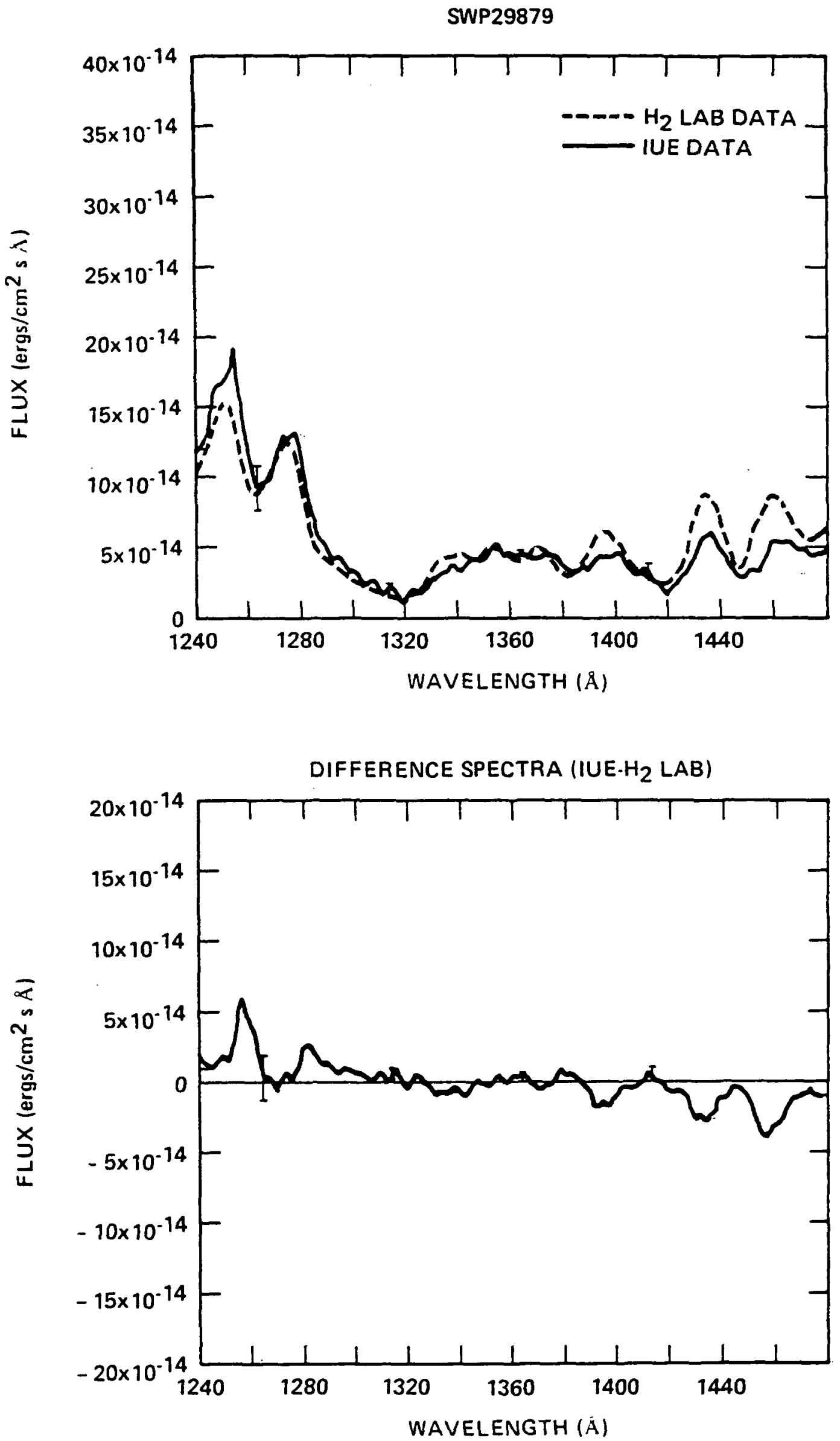

Fiqure 5 